\title{
Short communication: Dairy bedding type affects survival of Prototheca in vitro
}

\author{
N. Adhikari, H. E. Bonaiuto, and A. B. Lichtenwalner ${ }^{1}$ \\ Department of Animal and Veterinary Science, University of Maine, Orono 04469
}

\section{ABSTRACT}

Protothecae are algal pathogens, capable of causing bovine mastitis, that are unresponsive to treatment; they are believed to have an environmental reservoir. The role of bedding management in control of protothecal mastitis has not been studied. The purpose of this study was to evaluate the growth of either environmental or mastitis-associated Prototheca genotypes in dairy bedding materials that are commonly used in Maine. Prototheca zopfii genotypes 1 and 2 (gt1 and gt2) were inoculated into sterile broth only (control), kiln-dried spruce shavings, "green" hemlock sawdust, sand, or processed manure-pack beddings with broth, and incubated for $2 \mathrm{~d}$. Fifty microliters of each isolate was then cultured onto plates and the resulting colonies counted at 24 and $48 \mathrm{~h}$ postinoculation. Shavings were associated with significantly less total Prototheca growth than other bedding types. Growth of P. zopfii gt1 was significantly higher than that of $g t 2$ in the manure-pack bedding material. Spruce shavings, compared with manure, sand, or sawdust, may be a good bedding type to prevent growth of Prototheca. Based on these in vitro findings, bedding type may affect Prototheca infection of cattle in vivo.

Key words: bovine mastitis, protothecosis, Prototheca zopfii, bedding

\section{Short Communication}

Prototheca are unicellular yeast-like algae capable of causing infections in mammals. Protothecosis in cattle is usually caused by Prototheca zopfii (Lassa et al., 2011) and occasionally Prototheca wickerhamii (Lopes et al., 2008). Recently, an additional species, Prototheca blaschkeae, was identified as a bovine mastitis pathogen (Marques et al., 2008). Prototheca zopfii has 2 genotypes: gt1 and gt2, P. zopfii gt2 being the principal mastitis agent (Roesler et al., 2006; Möller et al., 2007; Ito et al., 2011; Sobukawa et al., 2011).

Received March 7, 2013.

Accepted August 22, 2013.

${ }^{1}$ Corresponding author: anne.lichtenwalner@maine.edu
Protothecosis can be clinical or subclinical (Lass-Flörl and Mayr, 2007) and can cause either acute inflammatory or chronic granulomatous mastitis (Lopes et al., 2008). Protothecal mastitis usually is associated with reduced milk yield (Lopes et al., 2008; Ito et al., 2011; Lassa et al., 2011) and can elevate the SCC (Ito et al., 2011; Lassa et al., 2011). Treating cattle for protothecal mastitis thought to be ineffective (Sobukawa et al., 2011). Standard mastitis treatments designed to kill bacterial pathogens do not eradicate Prototheca spp. from infected cows (Melville et al., 1999; Lassa et al., 2011). Currently, the only dependably effective control is elimination of the infected animals from the herd (Melville et al., 1999; Lopes et al., 2008; Lassa et al., 2011). Because of the expense of replacement animals and lost production opportunities, prevention of protothecal mastitis is the most desirable goal for dairy farmers. When protothecal mastitis is present, it may become widespread on the farm (Costa et al., 1997; Scaccabarozzi et al., 2008) and interventions such as changing bedding types may become cost effective compared with the cost of replacing lactating cows.

Prototheca spp. have been reported to grow well in a humid environment, especially in the presence of complex organic matter (Melville et al., 2002), such as damp areas contaminated with manure (Anderson and Walker, 1988; Costa et al., 1997). In addition, Prototheca can be shed in the manure of healthy cattle. Not surprisingly, therefore, P. zopfii can be found throughout the dairy environment, particularly in the mud around dairy barns (Costa et al., 1997). Prototheca zopfii gt1 has been reported to be more predominant in the environment than $P$. zopfii gt2, but it is not thought to cause mastitis. However, experimental infection of cattle with P. zopfii gt1 has been associated with granulomatous lesions in the mammary gland (Ito et al., 2011).

Because the route of protothecal infection is thought to be via invasion of the teat canal, exposure of the udder to bedding contaminated with Prototheca may increase the risk of infection. Even low protothecal colony counts may be problematic, because experimental intramammary inoculation of 40 to $480 \mathrm{cfu}$ of $P$. zopfii isolated from a naturally infected cow was ca- 
pable of causing mild mastitis (increased gland firmness and clots in the milk; McDonald et al., 1984). If even small numbers of Prototheca can cause mastitis, the importance of bedding types and other management practices cannot be neglected.

The choice of bedding for freestall pens or loafing areas could theoretically influence the risk of protothecal infection by reducing survival of this organism in contaminated bedding. In light of the possibility that Prototheca may infect the udder by contact with contaminated bedding materials in loafing pens, the purpose of this study was to evaluate the growth of $P$. zopfii gt1 and gt2 in bedding materials that are commonly used in the state of Maine.

An isolate of the known mastitis pathogen P. zopfii gt2 (SAG 2064 type strain) was compared with an isolate not known to be associated with naturally occurring mastitis, P. zopfii gt1 (SAG type strain 2063, isolated from pig slurry); both isolates were a gift from Uwe Roesler (Institute of Animal Hygiene and Veterinary Public Health, Faculty of Veterinary Medicine, University of Leipzig, Leipzig, Germany). Isolates were initially cultured at $26^{\circ} \mathrm{C}$ for $48 \mathrm{~h}$ in air using Sabouraud-dextrose agar at the University of Maine Animal Health Laboratory (Orono) from cryobeadpreserved strains stored at $-80^{\circ} \mathrm{C}$. A cell suspension was made from fresh cultures in $0.85 \%$ sterile saline with $0.01 \%$ Tween (Polysorbate 80, a nonionic surfactant used to reduce clumping), and adjusted to be equivalent to $47 \%$ transmittance $(\mathbf{T})$ at $590 \mathrm{~nm}$ (spectrophotometer model 21907; Biolog Inc., Hayward CA). Previous work in our laboratory established that the concentration of Prototheca at $47 \%$ T was $1 \times 10^{6} / \mathrm{mL}$. Each cell suspension was vortexed immediately before inoculation into the bedding or control bags.

Four bedding types were tested: kiln-dried spruce shavings, "green" (not kiln dried) hemlock sawdust, processed manure-pack, and sand. A control for bedding type (broth only) was included. Bedding materials were obtained from local sources. The kiln-dried spruce shavings were from Pleasant River Lumber (Enfield, ME); the sawdust was "green" hemlock sawdust from Chester Forest Products (Lincoln, ME); the bovine manure pack was a byproduct of a local dairy's energy generating facility, and the sand was obtained from the Facilities Management site at the University of Maine (Orono).

The bedding samples were obtained using random sampling from several sites in large (truckload) piles of shavings, sawdust, or sand. Randomization of the shavings, sawdust, and sand beddings was achieved by selecting 3 general areas thought to represent the whole pile [top, approximate middle ( $1 \mathrm{~m}$ deep), and side edges] and taking a sample of approximately $1 \mathrm{~L}$ from each. The manure bedding was provided by the producer in a gallon aliquot. Each bedding type was thoroughly mixed (by massaging the bag) before autoclaving. Each bag of bedding was then thoroughly remixed after autoclaving, before each subsample was removed from the bag. Thus, samples were homogenized twice before subsampling. All beddings were autoclaved using a dry cycle before use. To verify sterilization before use of the beddings in this trial, sterile dry cotton swabs were dragged through each sterilized bedding type, plated onto blood agar, incubated for $48 \mathrm{~h}$ at $37^{\circ} \mathrm{C}$, and read.

For each bedding type following mixing, 3 subsamples were obtained using sterile technique, each of sufficient volume to fill approximately $25 \%(125 \mathrm{~mL})$ of a sample bag. Weights were recorded after filling bags but before adding the broth or inoculum. Because of the large variation in density of bedding types, and the decision to hold protothecal inoculum and broth volumes constant, volume rather than weight was the primary unit of measure for bedding. One hundred milliliters of Prototheca isolation medium (Pore, 1973) without 5-fluorocytosine was added to each bag along with 1 $\mathrm{mL}$ of sterile saline (control), $1 \mathrm{~mL}$ of $47 \% \mathrm{~T} \mathrm{P}$. zopfii gt1, or $1 \mathrm{~mL}$ of $47 \%$ OD P. zopfii gt2 (treatments). Due to the use of sterilized bedding materials, the bacterial growth inhibitor 5 -fluorocytosine was deleted from the Prototheca isolation medium broth. The bags were sealed, pummeled, and incubated in a shaking water bath at $26^{\circ} \mathrm{C}$. After $48 \mathrm{~h}$, each bag was massaged to mix the sample, and $100 \mu \mathrm{L}$ of liquid contents was pipetted into a sterile tube containing $900 \mu \mathrm{L}$ of $0.85 \%$ saline with $0.01 \%$ Tween, and vortexed. The sample was then further diluted to produce $10^{-2}$ and $10^{-3}$ dilutions. Each tube was vortexed before transferring 50 $\mu \mathrm{L}$ onto a Sabouraud-dextrose agar plate (Northeast Labs, Waterville, ME) and evenly distributing it across the agar using a sterile glass spreader. Plates were then incubated in air at $26^{\circ} \mathrm{C}$ for $48 \mathrm{~h}$. Colonies were observed at 24 and $48 \mathrm{~h}$, and the 48-h colony count was recorded $(\mathrm{cfu} / 50 \mu \mathrm{L})$. Because plates inoculated with $50 \mu \mathrm{L}$ of the $10^{-3}$ dilution did not yield many colonies, data from this dilution were not analyzed. The counts $(\mathrm{cfu} / 50 \mu \mathrm{L})$ of the $10^{-2}$ dilution were converted to colony-forming units per milliliter (multiplied by 2 $\times 10^{3}$ ) and log-transformed before analysis. An overall test for significance of isolate, bedding type, and the interaction of isolate and bedding type (2-way ANOVA) was followed by pairwise comparisons using Tukey's honestly significant difference (HSD) test. Normality of residuals was confirmed using the Shapiro-Wilk normality test (GraphPad Prism 6 for Windows, version 6.02, 2013; GraphPad Software, La Jolla, CA; R (R Core Team, 2013).

Bedding weights per sample were normally distributed. Beddings were utilized on a volume basis (Table 
1). No growth was seen in the cultures of sterilized bedding before Prototheca inoculation. The negative controls (sterile saline and bedding) produced no growth at 24 and $48 \mathrm{~h}$ of incubation for any bedding type. We observed an overall effect of bedding type and isolate by bedding type $(P<0.001)$. Growth of both Prototheca isolates was lower in the positive control (no bedding) and shavings compared with manure, sawdust, and sand $(P<0.001 ;$ Figure 1$)$. The growth of $g t 1$ was higher than that of $g t 2$ in manure $(P=0.001)$ but not different from that of $g t 2$ in sawdust, sand, or shavings.

Based on these results, bedding type had a significant effect on Prototheca growth in vitro, and this effect was associated with increased growth in manure, sawdust, and sand beddings. In contrast, kiln-dried spruce shavings may inhibit Prototheca growth. This overall effect was seen in both P. zopfii genotypes. Manure had the most discordant effect, appearing to facilitate growth of P. zopfii gt1 more than P. zopfii gt2.

We investigated $P$. zopfii gt1 and $g t 2$ in this experiment because both have been isolated from dairy barn environments. Some reports indicate that environmental isolates are more likely to be $g t 1$; however, most mastitis-associated isolates have been $g t 2$ (Osumi et al.,

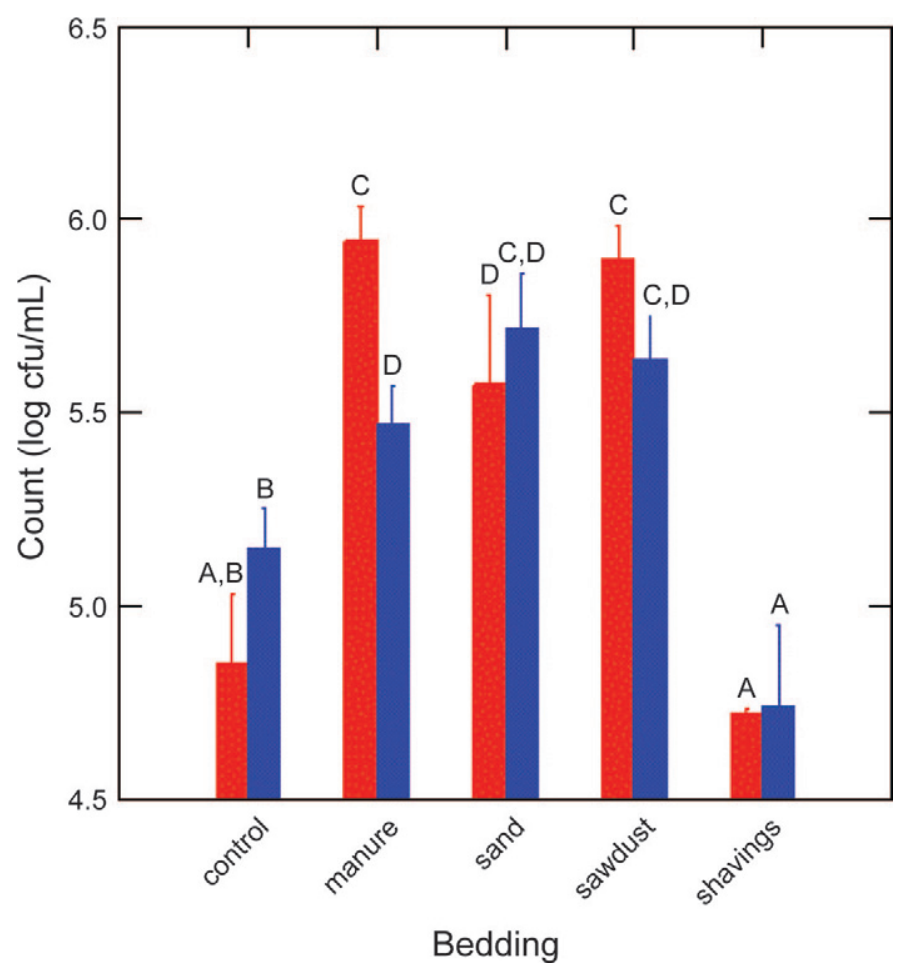

Figure 1. Effect of bedding type and Prototheca isolate on growth in vitro. Log-transformed counts (cfu) for Prototheca zopfii genotype 1 (gt1) and P. zopfii genotype 2 (gt2) in control (broth only), manure, sand, sawdust or shavings bedding substrates are shown. ${ }^{\mathrm{A}-\mathrm{D}}$ Bars with different letters are significantly different; $P<0.05$. Color version available in the online PDF.
Table 1. Bedding sample weights (mean $\pm \mathrm{SD}$ ) before adding broth and inoculum

\begin{tabular}{ll}
\hline Bedding type $^{1}$ & Weight $(\mathrm{g})$ \\
\hline Control (broth only) & - \\
Processed manure & $26.8 \pm 4.1$ \\
Sand & $66.6 \pm 12.0$ \\
Sawdust & $21.3 \pm 2.1$ \\
Shavings & $14.7 \pm 2.8$
\end{tabular}

${ }^{1}$ The volume of each sample was approximately $125 \mathrm{~mL}$.

2008). Prototheca zopfii gt2 has been found in dairy farm environments in Maine (A. Lichtenwalner, unpublished data). In addition, P. zopfii is not the only Prototheca species found in the dairy environment: $P$. wickerhamii has also been isolated on dairies (Anderson and Walker, 1988). The role of P. zopfii gt1 in the etiology of bovine mastitis is not clear; it has been hypothesized to be an environmentally adapted form of the more pathogenic gt2, based on experimental infection of the bovine mammary gland (Ito et al., 2011). If Prototheca is found in a dairy environment, it may be important for the farmer to know which species are present to evaluate the risk of protothecal mastitis.

More abundant growth of $P$. zopfii in manure-pack bedding was expected, because Prototheca in general appear to prefer damp areas contaminated with manure or other organic matter (Anderson and Walker, 1988; Costa et al., 1997). We expected the host-adapted gt2 to grow significantly better in manure bedding than the more environmentally adapted $g t 1$, but the opposite was the case. This finding might be expected, however, given that manure is an ever-present factor in the dairy environment.

Nonpotable water and damp areas have been suggested as environmental sources of Prototheca (McDonald et al., 1984). Retention of fluid in the bedding may foster the growth of Prototheca on the farm, but this effect was not evaluated in our study due to the use of a broth incubation medium before quantitative plating of the bedding samples. However, the effect of bedding either in retaining water or in facilitating drainage away from the recumbent cow may be important in either facilitating or inhibiting environmental survival of Prototheca.

The effects of $\mathrm{pH}$, relatively larger surface area, or relative content of metabolic substrates for Prototheca were not evaluated in this study. The presence of any substrate used in this experiment, with the exception of shavings, increased protothecal growth over the brothonly control. As the relative surface area should be lower in equal volumes of shavings versus sand, surface area may have been a major factor in this experiment. Sand, which would be unlikely to provide nutrients to Prototheca, may have functioned primarily as a sub- 
strate in this study. Inhibition was not directly tested in this experiment, and further work to test whether an inhibitory substance is present in shavings is needed.

Prototheca spp. have been shown to be resistant to many antibiotics and antifungals (Jagielski et al., 2012) and are capable of surviving a wide range of environmental conditions as well as disinfectants (Marques et al., 2010; Salerno et al., 2010; Lassa et al., 2011; Sobukawa et al., 2011). Within the host, protothecal cell walls are relatively resistant to enzymatic degradation probably due to their high sporopollenin content (Cheville et al., 1984). This persistence may contribute to spread within the host and to intermittent shedding of the organism in mastitis cases.

In general, protothecal mastitis is thought to be increasing in incidence and it is gaining more economic and public health importance (Marques et al., 2008). The difficulty of effectively treating protothecal mastitis supports the use of effective preventive measures on dairies at risk of protothecal mastitis, both in the milking parlor (Marques et al., 2010) and in the cow's environment. Utilization of bedding types that do not enhance protothecal growth may reduce entry of this pathogen into the udder. The cost-benefit will differ depending on many factors, including the price of bedding and the risk of protothecal mastitis on a particular farm. Although bedding has not been identified as a risk factor for protothecal mastitis, it may prove to be a useful tool in managing risk on at-risk dairies (Pieper et al., 2012).

\section{ACKNOWLEDGMENTS}

This work is based upon research supported in part by Hatch Grant number ME0- L-5-00514-13 (NE1048) from the USDA National Institute of Food and Agriculture (Washington, DC). This is Maine Agricultural and Forestry Experiment Station Publication \#3315.

\section{REFERENCES}

Anderson, K. L., and R. L. Walker. 1988. Sources of Prototheca sp. in a dairy herd environment. J. Am. Vet. Med. Assoc. 193:553-556.

Cheville, N., J. McDonald, and J. Richard. 1984. Ultrastructure of Prototheca zopfii in bovine granulomatous mastitis. Vet. Pathol. 21:341-348.

Costa, E., P. Melville, A. Ribeiro, E. Watanabe, and M. Parolari. 1997. Epidemiologic study of environmental sources in a Prototheca zopfii outbreak of bovine mastitis. Mycopathologia 137:33-36.

Ito, T., R. Kano, H. Sobukawa, J. Ogawa, Y. Honda, Y. Hosoi, H. Shibuya, T. Sato, A. Hasegawa, and H. Kamata. 2011. Experimental infection of bovine mammary gland with Prototheca zopfii genotype 1. J. Vet. Med. Sci. 73:117-119.

Jagielski, T., P. Buzzini, H. Lassa, E. Malinowski, E. Branda, B. Turchetti, A. Polleichtner, U. Roesler, P. Lagneau, S. Marques, E.
Silva, G. Thompson, R. Stachowiak, and J. Bielecki. 2012. Multicentre Etest evaluation of in vitro activity of conventional antifungal drugs against European bovine mastitis Prototheca spp. isolates. J. Antimicrob. Chemother. 67:1945-1947.

Lass-Flörl, C., and A. Mayr. 2007. Human protothecosis. Clin. Microbiol. Rev. 20:230-242.

Lassa, H., T. Jagielski, and E. Malinowski. 2011. Effect of different heat treatments and disinfectants on the survival of Prototheca zopfii. Mycopathologia 171:177-182. http://dx.doi.org/10.1007/ s11046-010-9365-7.

Lopes, M. M., R. Ribeiro, D. Carvalho, and G. Freitas. 2008. In vitro antimicrobial susceptibility of Prototheca spp. isolated from bovine mastitis in a Portugal dairy herd. J. Mycol. Med. 18:205-209. http://dx.doi.org/10.1016/j.mycmed.2008.09.001.

Marques, S., E. Silva, J. Carvalheira, and G. Thompson. 2010. In vitro susceptibility of Prototheca to $\mathrm{pH}$ and salt concentration. Mycopathologia 169:297-302.

Marques, S., E. Silva, C. Kraft, J. Carvalheira, A. Videira, V. A. Huss, and G. Thompson. 2008. Bovine mastitis associated with Prototheca blaschkeae. J. Clin. Microbiol. 46:1941-1945. http://dx.doi. org/10.1128/JCM.00323-08.

McDonald, J., J. Richard, and N. Cheville. 1984. Natural and experimental bovine intramammary infection with Prototheca zopfii. Am. J. Vet. Res. 45:592-595.

Melville, P. A., N. R. Benites, I. L. Sinhorini, and E. O. Costa. 2002. Susceptibility and features of the ultrastructure of Prototheca zopfii following exposure to copper sulphate, silver nitrate and chlorexidine. Mycopathologia 156:1-7.

Melville, P. A., E. T. Watanabe, N. R. Benites, A. R. Ribeiro, J. A. B. Silva, F. G. Junior, and E. O. Costa. 1999. Evaluation of the susceptibility of Prototheca zopfii to milk pasteurization. Mycopathologia 146:79-82.

Möller, A., U. Truyen, and U. Roesler. 2007. Prototheca zopfii genotype 2: The causative agent of bovine protothecal mastitis? Vet. Microbiol. 120:370-374.

Osumi, T., Y. Kishimoto, R. Kano, H. Maruyama, M. Onozaki, K. Makimura, T. Ito, and A. Hasegawa. 2008. Prototheca zopfii genotypes isolated from cow barns and bovine mastitis in Japan. Vet. Microbiol. 131:419-423.

Pieper, L., A. Godkin, U. Roesler, A. Polleichtner, D. Slavic, K. E. Leslie, and D. F. Kelton. 2012. Herd characteristics and cow-level factors associated with Prototheca mastitis on dairy farms in Ontario, Canada. J. Dairy Sci. 95:5635-5644.

Pore, R. 1973. Selective medium for the isolation of Prototheca. Appl. Microbiol. 26:648-649.

R Core Team. 2013. R: A language and environment for statistical computing. http://www.R-project.org/. R Foundation for Statistical Computing, Vienna, Austria.

Roesler, U., A. Möller, A. Hensel, D. Daumann, and U. Truyen. 2006. Diversity within the current algal species Prototheca zopfii: A proposal for two Prototheca zopfii genotypes and description of a novel species, Prototheca blaschkeae sp. nov. Int. J. Syst. Evol. Microbiol. 56:1419-1425.

Salerno, T., M. G. Ribeiro, H. Langoni, A. K. Siqueira, E. O. Costa, P. A. Melville, V. F. F. Bueno, A. A. M. Yamamura, U. Roesler, and A. V. da Silva. 2010. In vitro algaecide effect of sodium hypochlorite and iodine based antiseptics on Prototheca zopfii strains isolated from bovine milk. Res. Vet. Sci. 88:211-213. http:// dx.doi.org/10.1016/j.rvsc.2009.08.001.

Scaccabarozzi, L., B. Turchetti, P. Buzzini, G. Pisoni, L. Bertocchi, N. Arrigoni, P. Boettcher, V. Bronzo, and P. Moroni. 2008. Short communication: Isolation of Prototheca species strains from environmental sources in dairy herds. J. Dairy Sci. 91:3474-3477.

Sobukawa, H., M. Watanabe, R. Kano, T. Ito, M. Onozaki, A. Hasegawa, and H. Kamata. 2011. In vitro algaecide effect of disinfectants on Prototheca zopfii genotypes 1 and 2. J. Vet. Med. Sci. $73: 1527-1529$. 